\title{
Research on the Detection Algorithm of Workpiece Surface Defects Based on Machine Vision
}

\author{
Yuntao Zhang, Xiaorong Chen and Yin Yi \\ University of science and technology (south campus), No.334 Jungong Road, Yangpu district, Shanghai, China
}

\begin{abstract}
It is important to evaluate the workpiece whether the surface is defective or not. Because of the shadow area due to light angle by taking pictures, false edges are obtained. It will lead to that the real edge will be mistaken for surface scratches. Based on region growing, this paper introduces a new algorithm which is proposed to remove the shadow region, After the Gauss filter, the real defect of the component surface is obtained by the threshold segmentation. The experiments show that this method is suitable for a variety of scenarios of component surface defect detection. And it has the characteristics of high efficiency, stability and high precision.
\end{abstract}

Keywords-region growing; remove the shadow; defect detection; threshold segmentation

\section{INTRODUCTION}

With the rapid development of science and technology, in order to improve the production efficiency and precision of workpieces, industrial automation was born. And in recent years, it is widely used in industrial production. At the same time, the importance of quality keeps increasing. Surface defect is a very important standard of workpiece quality, including scratches, pits, grease, burrs, cracks, etc. Because scratch often appears on surface, more and more research is done on scratch in recent years. For example, literature [1] puts forward a kind of automatic test system using the method of multiple area scan CCD imaging, geometric segmentation and data synthesis. Literature [2] realizes automatic detection of steel plate surface defects based on the uniform entropy theory of pattern recognition. Using extract ROI image geometric features to reduce the dimension of ROI area from multidimensional eigenvector dimension. In literature [3] artificial neural network is applied to forecast the features of the workpiece surface quality, and the genetic algorithm is put to use optimize the structure of artificial neural network, the experiment show that the improved artificial neural network is not only suitable for predicting the characteristics of workpiece surface quality, But also the results are accurate. However these research methods often have a high requirement on the quality of the image acquisition, and in Multi-scenario the quality of defect detection is bad, the accuracy is not high, even the result can appear error detection. and for images of the shadow, the defects of the surface is not detected, because the shadow will produce false edge, which lead to that the real edge is judged as defects.

In view of the above problems, based on region growing this paper proposes an algorithm of removing the shadow of the image, which avoids the bad influence of shadow edge for edge detection. by using the Gaussian filter, it makes the surface defects of grey value contrast with the surrounding gray deserves more obvious, then the threshold segmentation method was adopted to realize the workpiece surface defect detection.

\section{PRINCIPLE AND GOAL OF DETECTION}

\section{A. Detection Goal}

Image 1 is the original image that achieved by using coaxial parallel light mirror, the surface defects can clearly view, and there are some small defects using observation method is unable to check. The detection method in this paper can not only detect the obvious defect, but also detect tiny flaws and scratch with high accuracy, high stability. Due to the images of acquisition contain shaded area, resulting in that edge detection can produce false edge, that is to say the edge of the shadow is detected for edge, and the edge of the actual workpiece is defined to defect, so the image pretreatment is necessary before surface defects detection, this paper adopts the method based on region growing to accurately inhibit the effects of the shadow. So the goal of this article research is to remove the shadow and detect the brake pad defect.

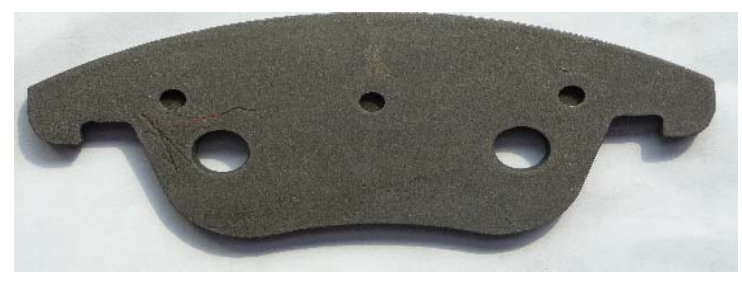

FIGURE I. ORIGINAL IMAGE

\section{B. Description of Detection Principle}

First of all, use coaxial parallel light mirror to achieve the image of brake pads, and then the collected image preprocessing is carried out, including image smoothing, image sharpening, median filtering, those methods primarily are used to reduce the bad effects of noise or some oily, at the same time image pretreatment also reduce the influence of gray value differences, which is produced by uneven gathering lamplight, secondly, use region growing algorithm (grating is $3 * 3$ ) to remove the shaded area from the image, and use Gaussian filtering smooth the image of no shadow. The aim is to reduce the influence of noise and enhance the robustness, meanwhile to enhance the gray of the surface defect contrast with the surrounding area. Finally extract the defects on the surface of 
the workpiece by using threshold segmentation, the flow chart is below:

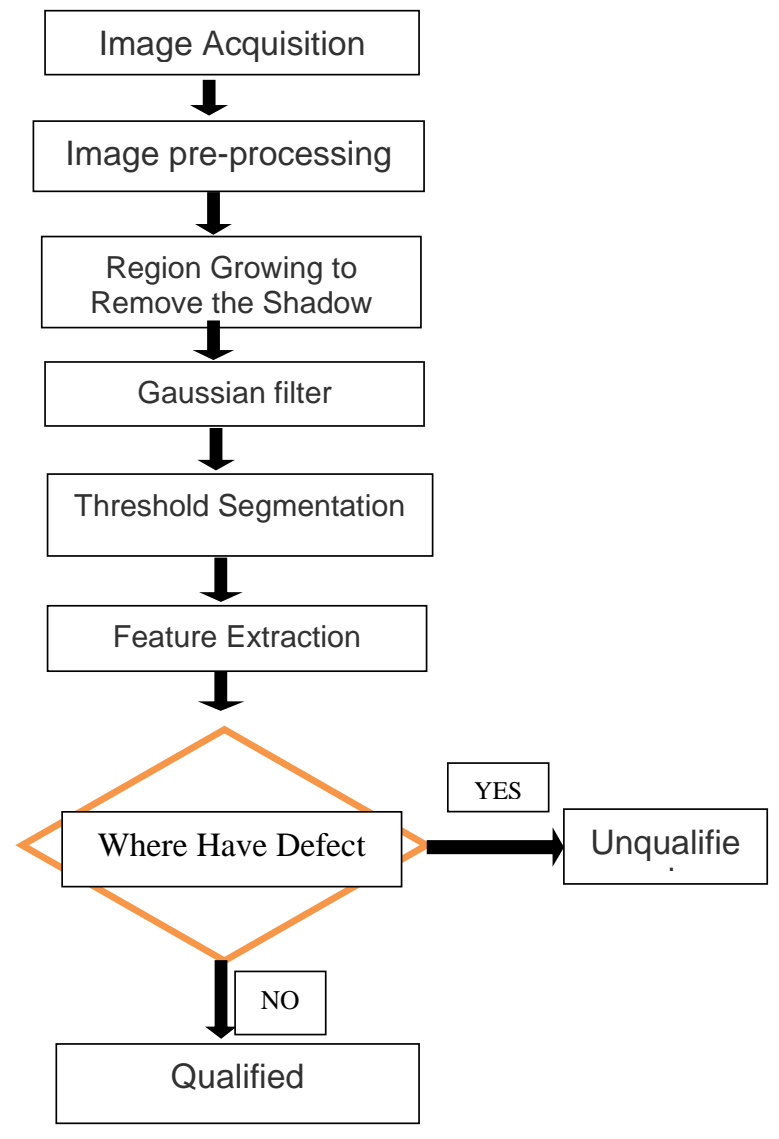

FIGURE II. FLOW CHART

\section{REMOVE THE SHADOW OF THE IMAGE BASED ON REGION GROWING ALGORITHM}

The earliest region growing image segmentation algorithm is put forward by Levine in literature [4]. The method generally have two ways, one is first given a small or seeded region of the object that is segmented in the target object. Then the pixels around are constantly added to the seed region by certain rules eventually combine all the pixels representing the object as the purpose of an area. The other is first segmented image into a lot of regions with strong consistency, such as the small regions of the same pixel gray value. According to certain rules put small regional integration into large area, and achieve the goal of image segmentation. The basic idea of region growing is to constitute the area by qualitative similarity pixel together. To each need to separate area, find a seed pixel as the starting point of growth. Then, pixels with the same or similar nature in the neighborhood of the seed are merged into the region where the seed pixels are located. and these new pixel as new seed pixels to continue the above process, until there is no meet the conditions of the pixel can be included, a region is finally formed. In this paper, region growing method steps are as follows:
1. For progressive scan the image and find out there is no ownership of pixel.

2. Put this pixel as the center and check the pixel around it, that is to say put the neighborhood compared with it one by one, if gray difference less than a predetermined threshold, then merge them, this article selects the threshold value is 6 .

3. With the new merged pixel as the center, repeat step 2, until the area cannot be further expansion.

4. Back to step 1, continue to scan until no pixel can be found without attribution, and then the growth process is over

Through the processing of the region growing algorithm, which makes the gray scale difference between shadow area and the artifact itself more clearly, and then through the corrosion expansion algorithm to remove the shadow area.

Gray image’s expansion detection operator:

$$
G_{1}=[g(x, y) \oplus s(x, y)]-g(x, y)
$$

Gray image corrosion operator:

$$
G_{2}=g(x, y)-[g(x, y) \Theta s(x, y)]
$$

$$
g(x, y) \text { is the image that is to be processed, } s(x, y) \text { is }
$$
ROI.

Region growing is the expansion and the corrosion process, expansion operator is to expand the image brighter than surround part , and shrink the back, it also can connect the bright objects in the grey images together with disconnect parts, and then divided the dark object. Corrosion operation is used to shrink prospects and expand background, it also can be used to separate interconnected bright objects and connect fragmented dark objects. Removal of the shadow results based on the region growing method is as follows:

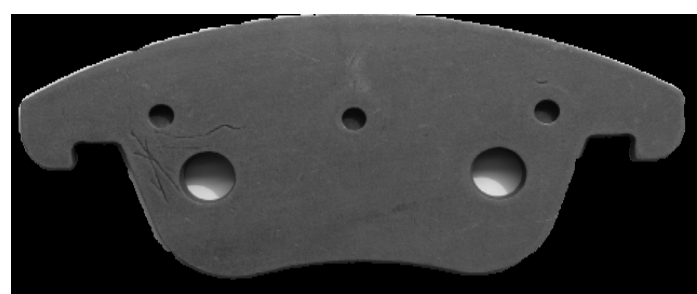

FIGURE III. REGION GROWING TO REMOVE THE SHADOW

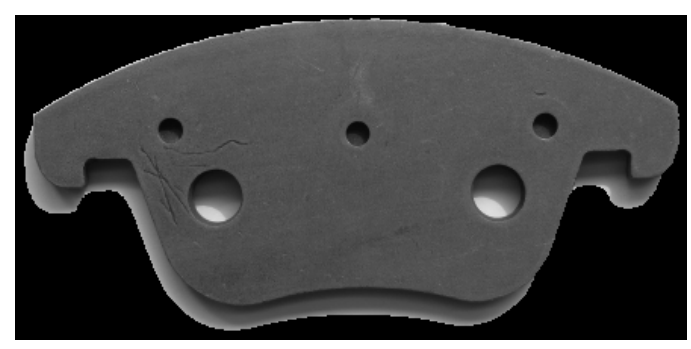

FIGURE IV. HAVE SHADOW 


\section{Surface Defect Detection Algorithm}

After image preprocess and region growing algorithm process, we can get the no shadow area of image, Use Gaussian filtering processing of image on this basis, Gaussian filter is a linear smoothing filtering, which is applicable to eliminate the Gaussian noise, Gaussian filtering is also a process to the weighted average of the whole image, each pixel value is achieved by weighted average of its own and other pixels within the neighborhood. Gaussian filtering concrete operation is: use a template (or convolution) to scan the each pixel in image, and the value of the center pixel of the template is replaced by using the weighted average grey value of pixels in neighborhood which is determined by templates This paper chooses the template for $3 * 3$ to enhance the grey value contrast between the surface defects and the surrounding. The results of Gaussian filtering processing are as follows.

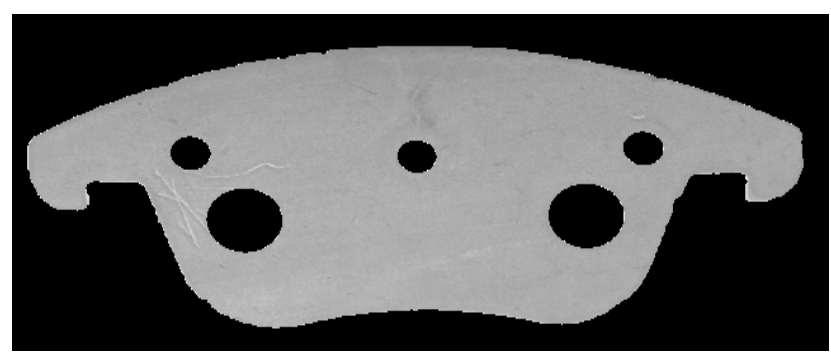

FIGURE V. GAUSSIAN FILTERING RESULTS

Gaussian filtering is not only for getting more clear defects image, but also acting as the reference image for threshold segmentation algorithm, images which are obtained by Gaussian filter, it can be seen that defect area of grey value is more obvious than the surrounding, and through Gaussian filtering the tiny defects and scratches of the surface can be achieved easily, which can improve the precision of the surface defect detection and accuracy. So adopt the image which is achieved by Gaussian filter processing as the input image when using threshold segmentation, this article selects the upper limit of threshold of 50 to extract defect features.

The threshold segmentation algorithm this article used is as follows:

$$
S=\left\{(r, c) \in R \| f_{r, c}-g_{r, c} \mid>g_{a b s}\right\}
$$

There $S$ is the result of threshold segmentation, $f_{r, c}$ is the result of removing the shadow, $g_{r, c}$ is the result of Gaussian filtering, $\mathrm{g}_{a b s}$ is the gray value compensation.

Such threshold segmentation method is very strict with the object shape, in order to make this method to get more extensive application, more stable and more accurate detection of defects on the surface of the work, taking into account the image grey value deviation when threshold segmentation, denote the permissible variations in the image by $\mathrm{V}_{r, c}$.

$$
S^{\prime}=\left\{(r, c) \in R \| f_{r, c}-g_{r, c} \mid>\mathrm{V}_{r, c}\right\}
$$

The $S^{\prime}$ is the result of segmenting the pixels that differ from the reference image by more than the permissible variation, the $\mathrm{V}_{r, c}$ can be determined by learning them from a set of training images.

\section{The Results Analysis about Removing the SHadow OF SURFACE DEFECT DETECTION}

According to the above method. Under the development environment of Halcon. We can get the no shadow area of the image by the region growing algorithm for the collected images. As shown in the figure below, by analyzing the test results we know the algorithm based on region growing to remove the shadow has high precision and stability, and also has Strong ability to resist noise in this paper. Based on no shadow image, using the paper optimized the best threshold segmentation to detect defects on the surface, and then determine whether the surface of original images is flawed or not, Test results show that detection method removed the shadow of the artifact surface defect has the high practicability and accuracy, and it is capable of detecting the very small defects on the surface of the surface.

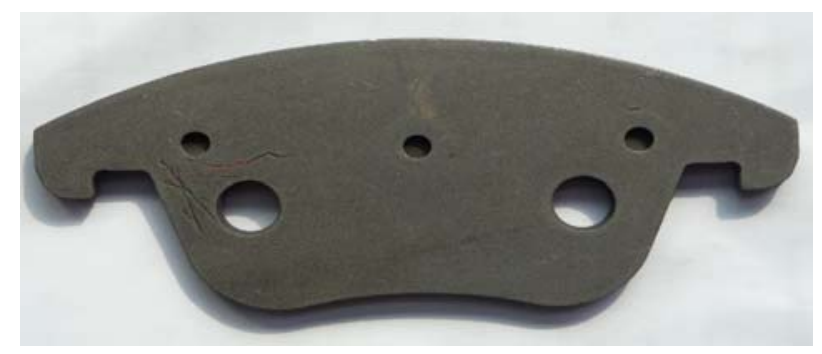

FIGURE VI. ORIGINAL IMAGE

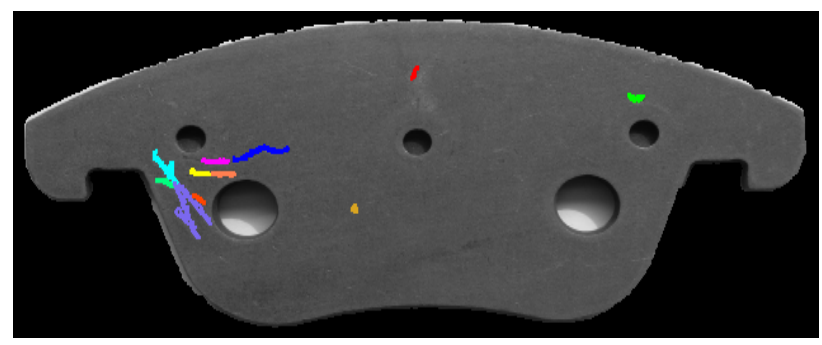

FIGURE VII. DEFECT DETECTION RESULTS

\section{CONCLUSION}

This paper solves a technical problem in the field of industrial automation, because of the development of industrial automation, high precision requirement is proposed for testing of different scenarios the shaded area is the main obstacle to this requirement, owing to the existence of artifacts shadow, which will lead to get the error edge and affect the subsequent feature extraction. Algorithm proposed in this paper is suitable for defect inspection of a variety of scenarios, and under different scenarios, the method can accurately get no shadow 
images, experiments show that this method is practical, strong stability and high precision.

\section{ACKNOWLEDGMENT}

Shanghai Key Laboratory of Modern Optical System \& Engineering Research Center of Optical Instruments and Systems, Ministry of Education; University of Shanghai for Science and Technology, School of Optical-Electrical and Computer Engineering, Shanghai, China.

\section{REFERENCES}

[1] Zhi-yong Luo, Dong-yu, Liu, Tao Jiang, etc. New online cold rolled steel strip surface defect inspection system [J]. Journal of Huan-zhong university of science and technology, 1996, 24 (1): $75 \sim 78$.

[2] Ke Xu etc. Automatic test system for cold rolled steel strip surface study [J]. Journal of iron and steel, 2000, 35 (10): 6.

[3] Yong-guang Chen, Guo-zhu Wang, Wang Yang etc. Wood surface defects edge shape detection algorithm research, wood processing machinery. 2003, (3): 18 to 22.

[4] Chun-ying Li. Machine vision in the application of steel ball surface defect detection [J]. Computer and modernization, 2005 (10): 63-65.

[5] Lin-hong Xu, Gui-mei Zhang, Shao-ping Chen. Engine surface defect detection based on machine vision technology [J]. Computer and modernization, 2010 (4): 156-162.

[6] Sheng-min, Luo.Edge detection algorithm based on improved mathematical morphology [J].Computer Simulation, 2011, 28(7); 301304.

[7] Yi Liu, Bing Huang, Huai-Jiang Sun, etc. Image segmentation based on visual saliency and graph cuts [J]. Computer-aided Design \& Computer Graphics, 2013, 25(3): 402-409

[8] Zhi Wang, Sai-Xian He. An adaptive edge detection method based on the theory of the Canny [J]. Chinese journal of image and graphics, 2004, 9 (8): 957-961. 